\title{
Selected CMS results in Higgs Physics
}

\author{
Milos Dordevic on behalf of the CMS Collaboration \\ Vinca Institute of Nuclear Sciences, University of Belgrade \\ E-mail: milos.dordevicecern.ch
}

\begin{abstract}
A selection of the most recent results of Higgs boson production and properties from the CMS Collaboration using the LHC Run 2 data is reported. The studies presented include analyses of Higgs boson decaying to two photons, four leptons via $\mathrm{Z}$ boson pair and associated production with top quark pair, predicted within the Standard Model (SM) of particle physics. Outlined searches for physics beyond the SM are the inverted top coupling search using Higgs boson produced with single top quark, then charged Higgs boson decaying to tau and neutrino and MSSM Higgs boson decaying to tau pairs. Prospects for future searches for the SM Higgs boson at the High Luminosity LHC and upgraded CMS detector are reported at the end.
\end{abstract}

55th International Winter Meeting on Nuclear Physics

23-27 January, 2017

Bormio, Italy 


\section{Introduction}

After the discovery of the Higgs boson at CERN $[1,2]$ the focus is now on the measurement of his properties, precise studies of it's decays and couplings to other particles. The CMS Collaboration has studied a wide range of Higgs production and decay channels based on LHC Run 1 dataset and found all of them compatible with the SM prediction. No additional Higgs bosons were observed and a few moderate excesses have been reported ( $\mathrm{t} \mathrm{H} \rightarrow$ multilepton), all within the uncertainties. An excellent performance of the CMS detector [3] was achieved in 2016 and around $37.8 \mathrm{fb}^{-1}$ of data was recorded. New results of Higgs boson studies at the energy of $13 \mathrm{TeV}$ and highest available luminosities from the 2016 dataset are produced and presented in this overview.

\section{Higgs decay to two photons}

Higgs boson decays to photon pair is a rare process with a small branching ratio, but the CMS detector capabilities allow for reconstruction of the diphoton invariant mass, shown in Fig. 1, with a great precision. The analysis [4] has an extensive usage of the multivariate methods (BDT), first to select the diphoton vertex with recoiling tracks and conversions, then the BDT is used to identify the photon candidates and finally another BDT is trained to select the diphoton events. The events were classified including categories with two forward jets (VBF) and also with top quark pair, of which one top quark decays leptonically or both undergo hadronic decay. The invariant mass spectrum was fit with data driven background model using discrete profiling method. The observed and expected signal significance for the Higgs mass value of $125.09 \mathrm{GeV}$ [5] were found to be 5.6 and 6.2 standard deviations, respectively. Best fit of the combined signal strength was measured to be $0.95_{-0.18}^{+0.21}$ when the mass parameter is profiled in the fit and it is presented in Fig. 2. This new study at the record energy of $13 \mathrm{TeV}$ confirms the existance of Higgs boson via it's decay to photon pair, that was first observed in LHC Run 1.

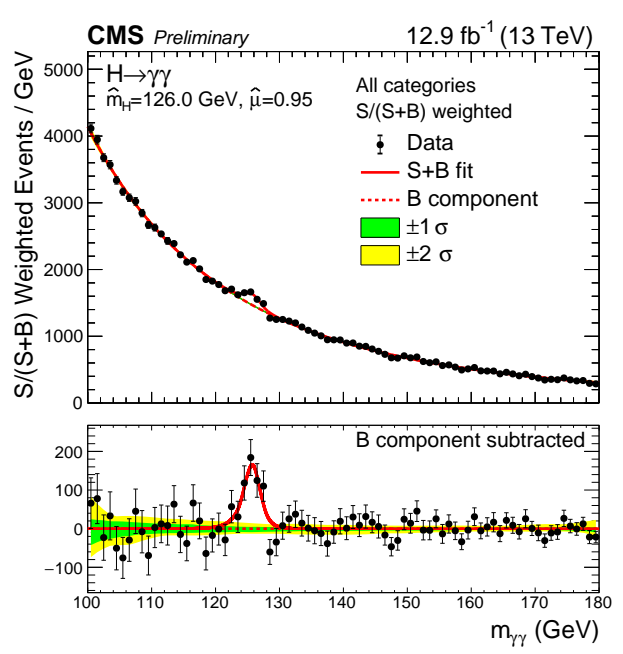

Figure 1: Data with signal plus background model fit for all categories summed weighted by their sensitivity [4].

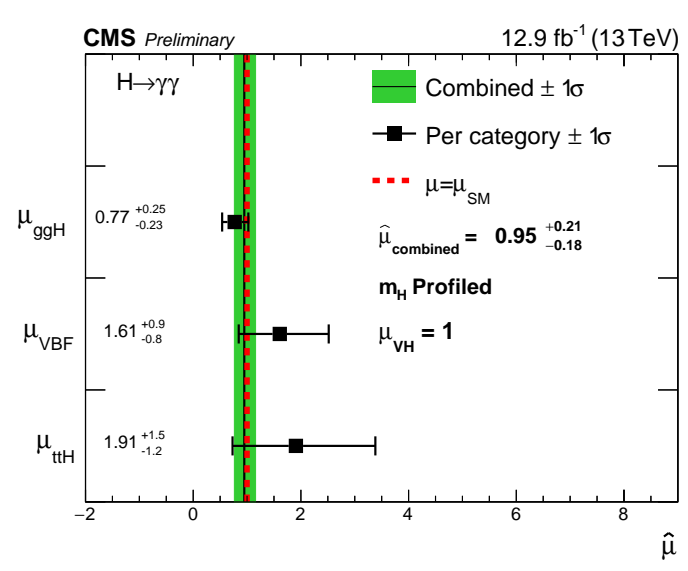

Figure 2: Signal strength modifiers for each process for profiled $m_{H}$, compared to SM expectation [4]. 


\section{Higgs decay to four leptons}

A clean experimental signature with a large signal to background ratio due to an excellent lepton momentum resolution, provides a possibility for a precise measurement of the Higgs boson decay to four leptons, either electrons or muons, via production of $Z$ boson pair and their subsequent decay to leptons. The four lepton invariant mass after the final event selection is shown in Fig. 3. The analysis [6] incorporates with respect to the previous iteration with LHC Run 1, new categories that target $\mathrm{VBF}, \mathrm{VH}$ and $\mathrm{ttH}$ production modes and standardly uses the matrix element kinematic method to discriminate the signal from a relatively small SM background. The Higgs boson mass was measured using this final state and the corresponding value of $124.50 \pm 0.47 \mathrm{GeV}$ was obtained. A rediscovery of the Higgs boson is achieved in this analysis, with the observed and expected signal significance of 6.5 and 6.2 standard deviations for the value of Higgs boson mass of $125.09 \mathrm{GeV}$, respectively. The fiducial cross section was measured at the $13 \mathrm{TeV}$, compared to the $7 \mathrm{TeV}$ and $8 \mathrm{TeV}$ results and found to be in a good agreement with the SM prediction, as shown in Fig. 4. A dedicated analysis was performed to measure the Higgs boson width using the off-shell method that is more precise than the on-shell and found it to be smaller than $41 \mathrm{MeV}$.
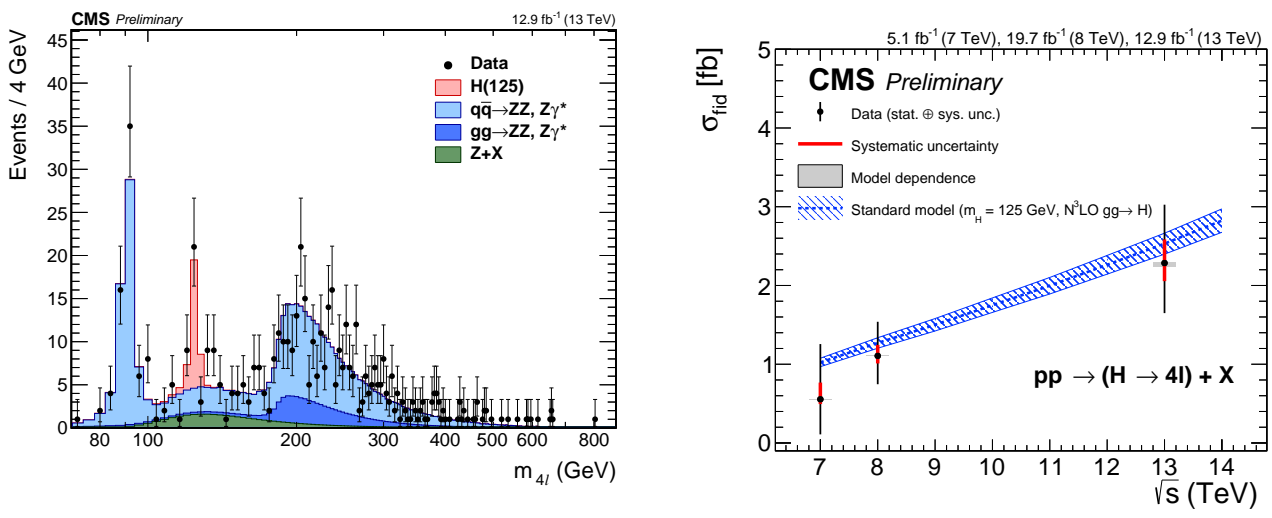

Figure 3: Distribution of the four-lepton reconstructed invariant mass $m_{4 l}$ in the full mass range [6] .

Figure 4: Measured fiducial cross section as a function of $\sqrt{s}[6]$.

\section{Higgs production with top quark pairs}

The Higgs boson production in the association to top quark pair $(\mathrm{ttH})$ is a rare process with a small cross section but the distinctive combination of Higgs boson decays paired with the decays of top quarks makes it experimentally accessible. Measurement of the ttH cross section allows for a direct access to the top-Higgs Yukawa coupling. The CMS Collaboration has repeated the measurements in all $\mathrm{ttH}$ final states from LHC Run 1 using the 2016 data at the energy of $13 \mathrm{TeV}$.

\subsection{Higgs decay to pair of b-quarks}

Two separate channels were considered in this analysis [7], with either one or two leptons (electrons or muons) produced with number of hadronic jets in the final state, that is based on the decays of the top quarks associated to Higgs boson. To enhance the signal sensitivity, the 
events were further categorised according to the number of jets and b-tagged jets. In each of these categories, the machine learning (BDT) and physics motivated (matrix element - MEM) methods, or their combination, are used to achieve the most optimal separation of the signal from the background processes. For example, in the category with two leptons and at least four jets of which all three are b-tagged, a cut on the BDT output is used to define a phase space for the application of the MEM discriminator and this is shown in Fig. 5. Simultaneous binned maximum likelihood fit to data in all categories is performed. The best-fit on the signal strength obtained is $-0.2 \pm 0.6$ for the value of Higgs mass of $125 \mathrm{GeV}$. The observed and expected limit at the 95 percent confidence level are 1.5 and 1.7, respectively, and this is shown for each category and their combination in Fig. 6.
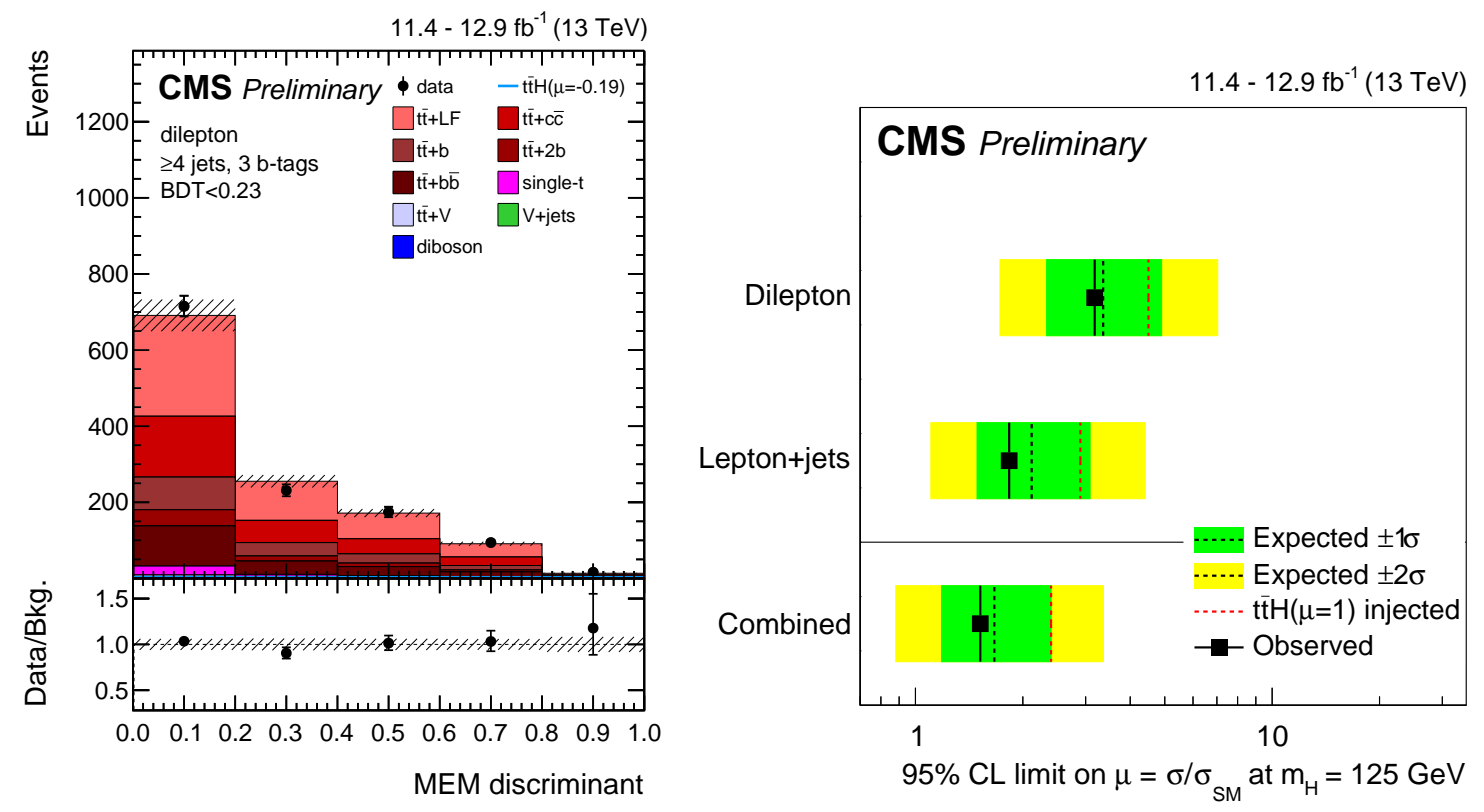

Figure 5: Final discriminant in the dilepton channel, for the analysis category with at least 4 jets, 3 b-tag jets and BDT score below 0.23 [7].

Figure 6: Median expected and observed $95 \%$ confidence level upper limit on the signal strength [7].

\subsection{Higgs decay to multileptons}

The decays of Higgs boson to pair of W bosons, Z bosons or tau leptons are considered in this analysis [8]. Two distinctive categories are made to enhance the presence of the signal: one with two same-sign leptons (electrons or muons) and the other with at least three leptons. In both categories also the number of (b-tagged) jets is selected. A dedicated BDT is trained to reject the non-prompt leptons, then leptons misidentified as jets and also the leptons with misidentified charge. Subcategories are defined with respect to the lepton flavor and charge, number of b-tagged jets and presence of hadronic taus in the event. Two BDTs are trained in order to maximize the presence of signal with respect to the $t \bar{t}$ and $t \bar{t} W / Z$ processes. The two-dimensional plane spanning the output of the two BDT trainings is divided into several bins and the signal and background content of each bin is folded to the one-dimensional histogram. The signal extraction is performed 
by applying a fit to the distribution of events among these bins. The Fig. 7 show the distribution of the output of two-dimensional BDT in the two same-sign category, after the final selection. The best fit of the signal strength for the combined categories is $2.0_{-0.7}^{+0.8}$, as shown in Fig. 8 and it is found to be in an agreement with the SM prediction.

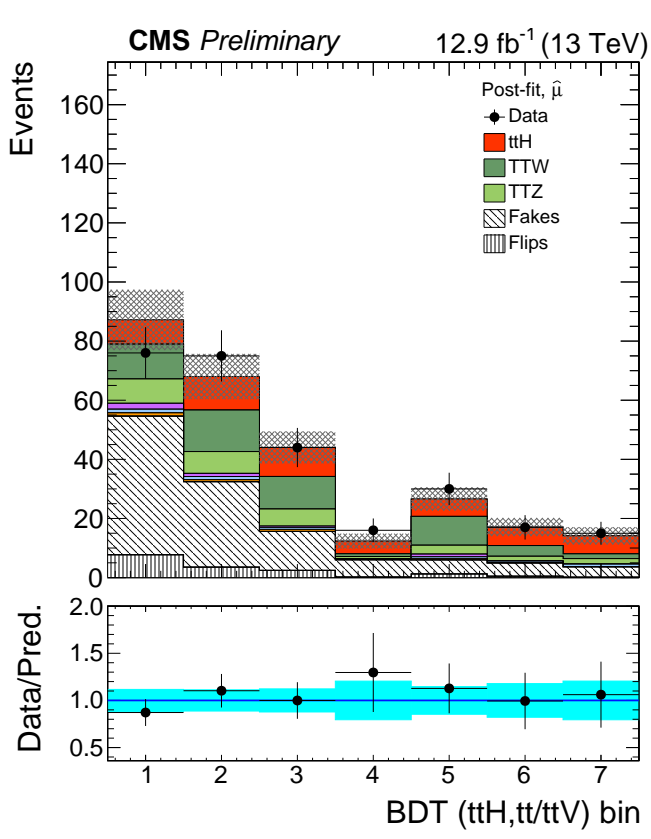

Figure 7: The BDT classifier output used for signal extraction in the same-sign dilepton channel [8].

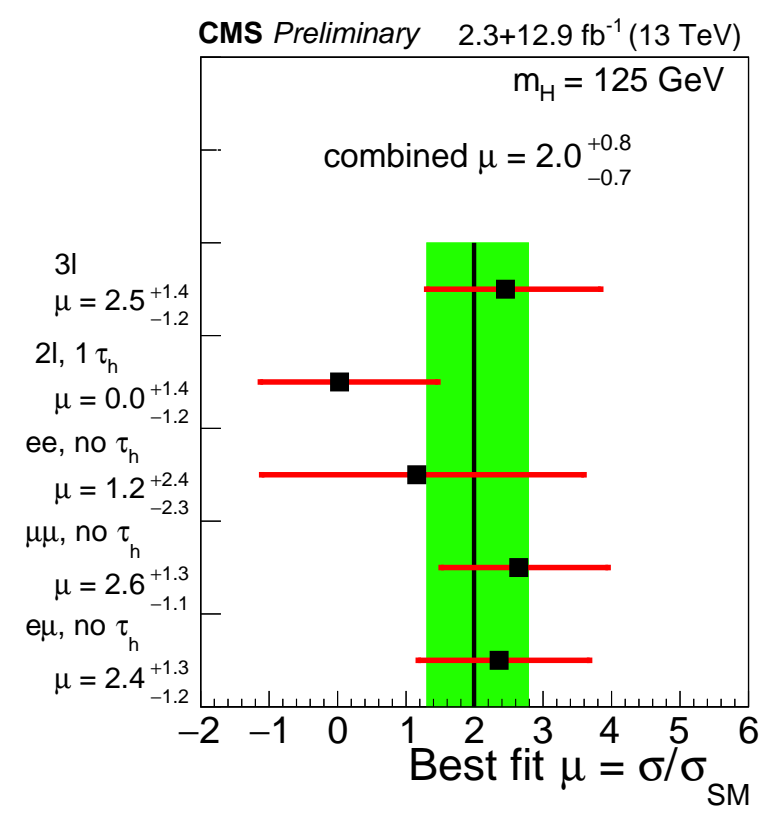

Figure 8: Best fit signal strength for the combined 2015+2016 analysis using 2.3 and $12.9 \mathrm{fb}^{-1}$, respectively, in the dilepton and trilepton channels and further split into flavor categories [8].

\subsection{Higgs decay to two photons}

This analysis [4] is a part of the more general study of Higgs decaying to two photons and requires additional pair of top quarks reconstructed in the event. The main backgrounds are the irreducible $t \bar{t}+\gamma \gamma$ and $t \bar{t}+\gamma+$ jet which is the reducible component and where the jets are misidentified as photons. One of the crucial aspects of the analysis is to suppress the mis-identified (fake) photons. The events are categorized according to the presence of at least one lepton ("leptonic tag") or no leptons are reconstructed at all ("hadronic tag"). At least two jets of which at least one b-tagged is required in the "leptonic tag" category, while at least five jets with at least one b-tagged are selected for the "hadronic tag" category. The signal extraction strategy is the same as in the inclusive $H \rightarrow \gamma \gamma$ analysis, where a diphoton mass resonance is being searched for on top of a non-resonant smoothly falling background. The analytic function is used to fit the signal events in each category and for each simulated Higgs mass point. Parametric fit functions with several functional forms, where a large set of function families are treated as a discrete parameter in a likelihood fit, are used to model the background. The data with signal plus background model fit are shown in Fig. 9 for the "leptonic" and Fig. 10 for "hadronic" tag category. The observed signal strength obtained by combining the two categories is $1.9_{-1.2}^{+1.5}$ for the $m_{H}=125.09 \mathrm{GeV}$. 


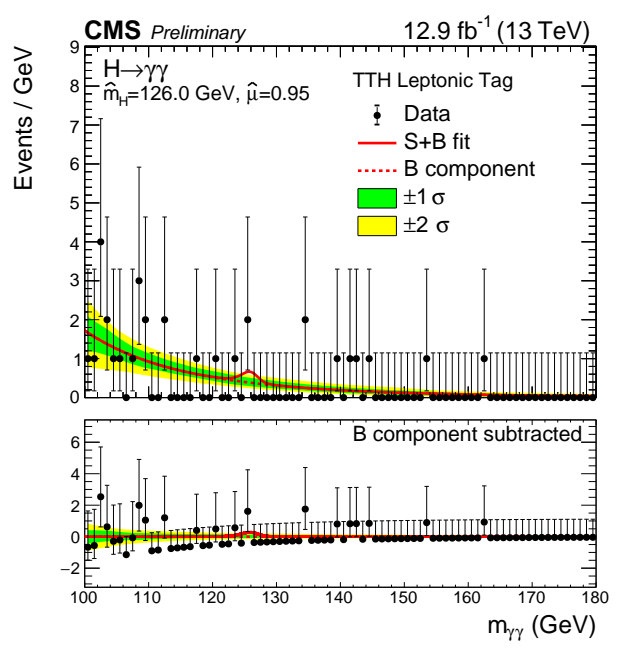

Figure 9: Data and signal plus background model fits in ttH category with the leptonic tag [4].

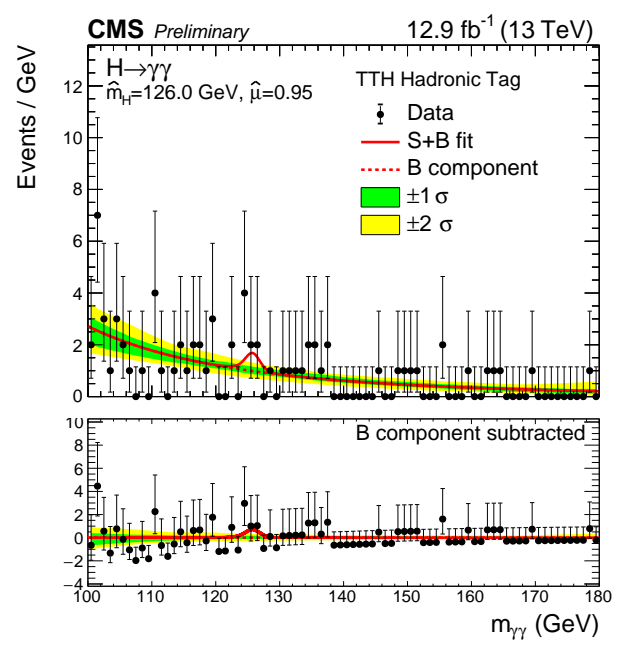

Figure 10: Data and signal plus background model fits in ttH category with the hadronic tag [4].

\section{Single top plus Higgs production}

The leading processes contributing to the single top plus Higgs ( $\mathrm{tH}$ ) production depend strongly on the $k_{t}$ and $k_{V}$ couplings. In this analysis [9] the dominant t-channel and tW-channel are considered. In the SM there is a destructive interference between these two channels, leading to a small cross section of about $90 \mathrm{fb}^{-1}$. The tH process can be used to measure the magnitude, but is also sensitive to the sign of top-Higgs Yukawa coupling since it's cross section depends strongly on the parameter $k_{t}$. If this parameter is negative, i.e. $k_{t}=-1$ then the cross section for this process is increased for about an order of magnitude. This scenario is referred as the inverted top coupling (ITC), which is not currently excluded if the BSM physics is allowed in the loops. The CMS Collaboration searched for $\mathrm{tH}$ production with Higgs boson decay to a pair of b-quarks and leptonic $\mathrm{W}$ decay from the top quark. The event categories with three or four b-tagged jets are defined. The event reconstruction related to proper assignment of the reconstructed b-quarks was applied, with different trainings per $\left(k_{t}, k_{V}\right)$ point. Another BDT was used for signal extraction, an example shown in Fig. 11. The SM tH production was excluded with observed and expected significance of 113.7 and 98.6 times SM prediction, respectively. The case of inverted top coupling has a sensitivity of 6.0 observed and 6.4 expected standard deviations, as shown in Fig. 12 and this is close to LHC Run 1 results even when using an order of magnitude less data.

\section{Charged Higgs decay to tau leptons}

The Two Higgs Doublet models (2HDM) predict a charged Higgs boson and CMS Collaboration has searched for the decay of this particle to tau and it's neutrino. The analysis [10] required to trigger on hadronic tau with transverse momentum larger than $50 \mathrm{GeV}$ and missing transverse energy larger than $90 \mathrm{GeV}$. The optimised event selection, corresponding to the maximum value of $S / \sqrt{B}$, requires at least three jets of which at least one is b-tagged. Dedicated kinematic require- 


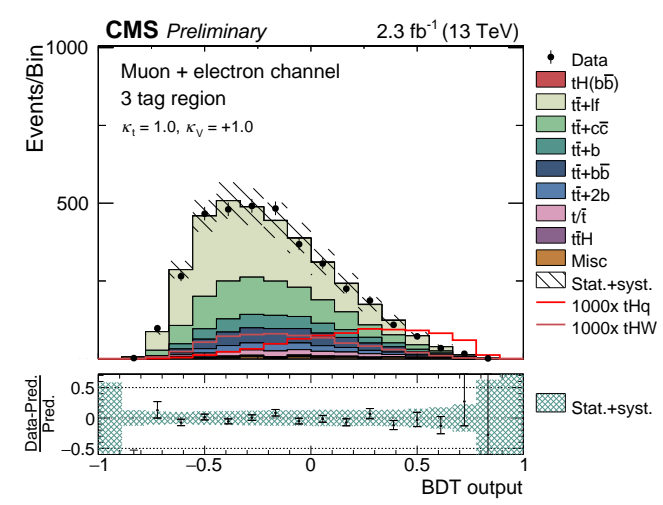

Figure 11: Postfit distribution of the classification BDT response in the 3 tag region for the SM and ITC coupling scenarios [9].

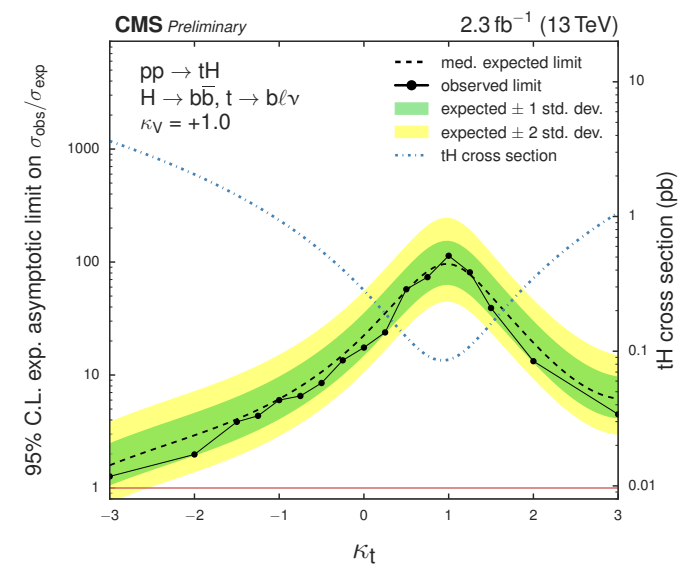

Figure 12: Upper limits on the tH scenario with $k_{V}=+1$, shown together with the tH cross section [9].

ments are imposed on the angular distributions between the reconstructed jets, hadronic tau and missing transverse energy. The signal is extracted using the transverse mass distribution, shown in Fig. 13. Model independent limits with respect to the mass of the charged Higgs boson are derived at the 95\% confidence level, as shown in Fig. 14. Also, exclusion limits in two dimensional plane spanned by mass of the charged Higgs boson and $\tan \beta$ for the $m_{h}^{\bmod +}$ scenario are set at the $95 \%$ confidence level.
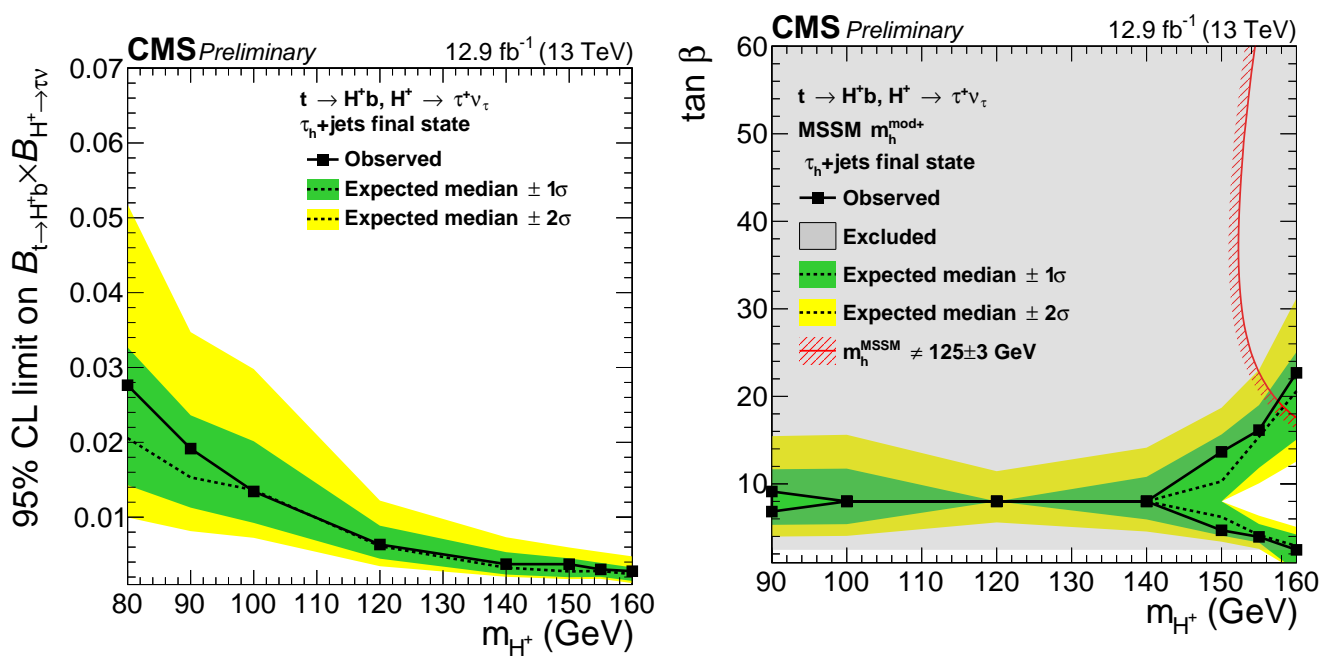

Figure 13: The expected and observed 95\% CL exclusion limits compared to the expectations from the SM [10].

Figure 14: The exclusion limits in the $m_{H^{ \pm}}-\tan \beta$ plane in the context of the $m_{h}^{\text {mod }+}$ model, for the low mass search [10].

\section{MSSM Higgs decay to tau pairs}

The Minimal Supersymmetric Standard Model (MSSM) Higgs boson is predicted to have an 
enhanced decay rate to taus, via the dominant gluon fusion production and a second dominant production mode that is the b-associated production. The analysis [11] performed by the CMS Collaboration considers four final states: $e \mu, e \tau_{h}, \mu \tau_{h}$ and $\tau_{h} \tau_{h}$. The total transverse mass, based on the missing transverse energy and visible mass of two tau leptons, is chosen as a variable most sensitive to the presence of the signal. The distribution of this variable is shown in Fig. 15. No significant excess was found on top of the SM expectation and model independent limits are set in the mass range of 90 to $3000 \mathrm{GeV}$, as shown in Fig. 16. Exclusion limits are also derived for the $m_{\phi}^{\bmod +}$ and MSSM scenarios as a function of $m_{A}$ and $\tan \beta$.

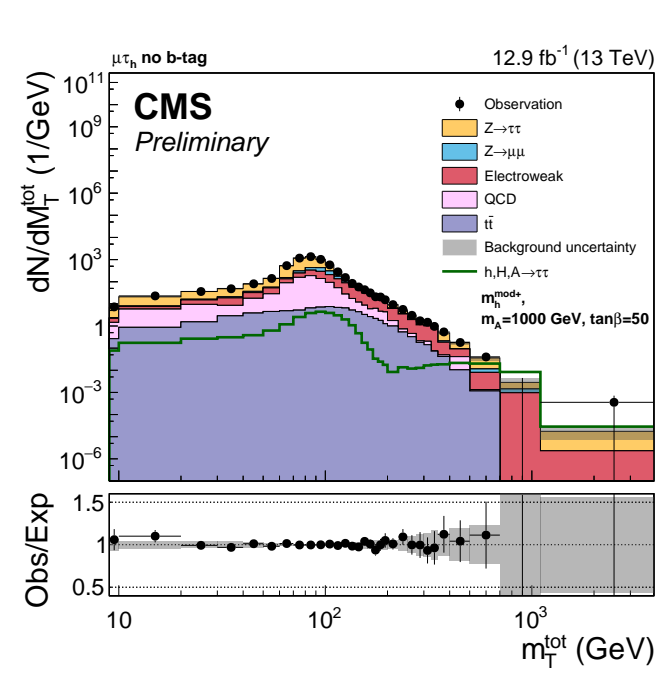

Figure 15: Post-fit plot of the total transverse mass distribution in the no b-tag category of the $\tau_{\text {had }} \tau_{\text {had }}$ channel [11].

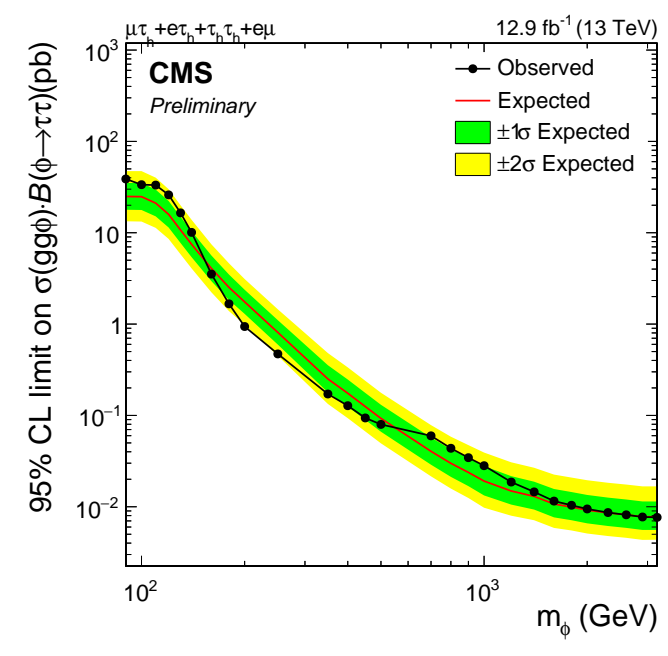

Figure 16: Expected and observed limits on cross-section times branching fraction for the gluon fusion process $(\mathrm{gg} \phi)$, resulting from the combination of all four channels [11].

\section{Future prospects for Higgs studies}

The extrapolation of results was made to give prediction of sensitivities for Higgs studies with larger datasets of $300 \mathrm{fb}^{-1}$ and $3000 \mathrm{fb}^{-1}$ and upgraded CMS detector for HL-LHC [12]. Scenarios with systematic uncertainties being kept constant with increasing luminosity and performance of CMS detector unchanged (S1), as well as taking into account high pileup conditions of HL-LHC and detector upgrades $(\mathrm{S} 1+)$ are considered. The scenarios S2 and S2+ where the theoretical uncertainties are scaled down by a factor of $1 / 2$, while systematic uncertainties scaled down by the square root of the integrated luminosity (until they reach lower limit set by the upgraded detector) are examined, for the nominal and upgraded CMS detector, respectively. For the Higgs to diphoton projection the analysis [4] was taken as a reference. At high pileup of 200 [13] it is expected that the vertex finding efficiency is degraded by $40 \%$, while the photon identification efficiency in the EB and EE is worse by 2.3 and 10 percent, respectively. The expected signal strength per production mode is presented in Fig. 17. The projection for Higgs to four lepton analysis at $3000 \mathrm{fb}^{-1}$, based on [6], is made taking into account different lepton efficiency and misidentification rates 
accounting for higher pileup at the HL-LHC. The expected signal strength per production mode in this channel is presented in Fig. 18.
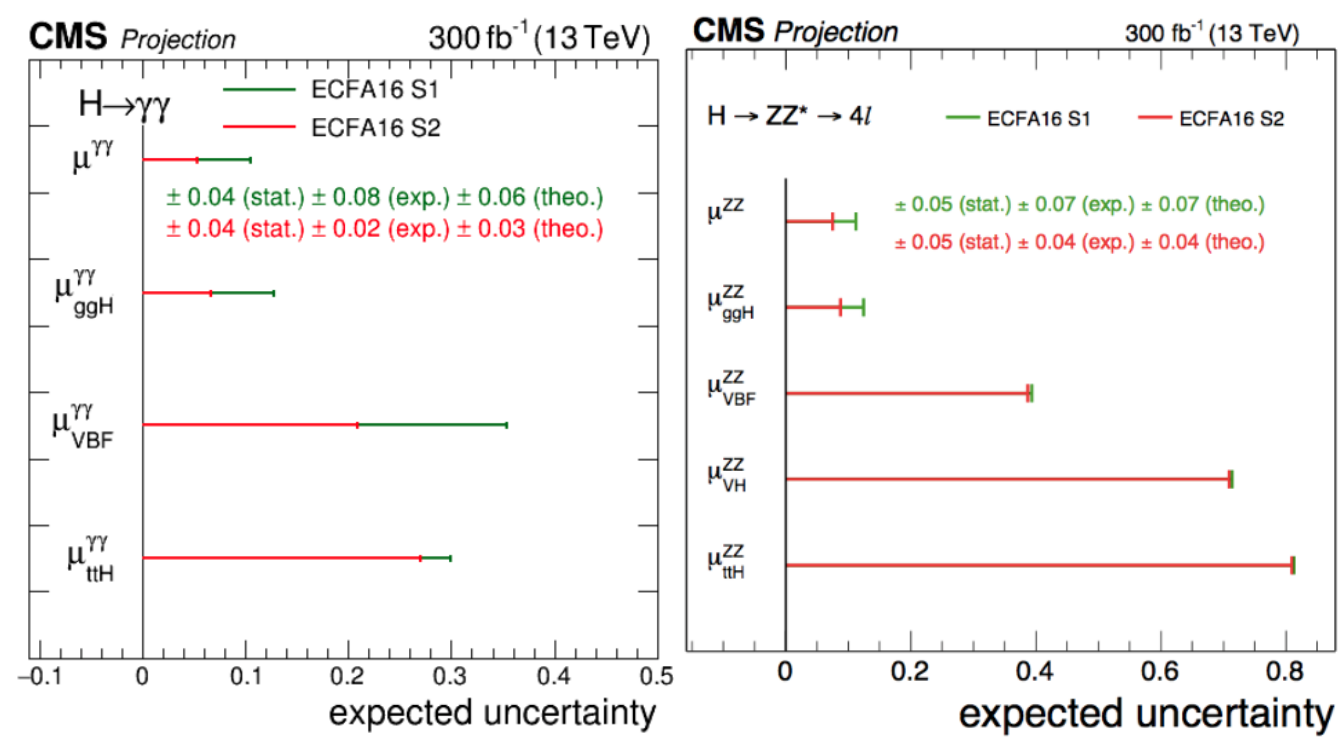

Figure 17: Expected uncertainty on the signal strength per production mode in

Figure 18: Expected uncertainty on the signal strength per production mode in Higgs to diphoton channel [12].

Higgs to four lepton channel [12].

\section{Conclusions}

The results using $12.9 \mathrm{fb}^{-1}$ of LHC Run 2 recorded by the CMS experiment in 2016 already confirm the existence of Higgs boson decaying to a pair of photons and four leptons via pair of $\mathrm{Z}$ bosons. No significant deviations from the SM prediction was found and consequently no extra Higgs bosons predicted by BSM theories discovered. Projections are made for HL-LHC scenarios.

\section{References}

[1] The ATLAS Collaboration, Phys. Lett. B 716, 1 (2012).

[2] The CMS Collaboration, Phys. Lett. B 716, 30 (2012).

[3] The CMS Collaboration, JINST 3 S08004 (2008).

[4] The CMS Collaboration, CMS-PAS-HIG-16-020 (2016).

[5] The ATLAS and CMS Collaborations, Phys. Rev. Lett. 114, 191803 (2015).

[6] The CMS Collaboration, CMS-PAS-HIG-16-033 (2016).

[7] The CMS Collaboration, CMS-PAS-HIG-16-038 (2016).

[8] The CMS Collaboration, CMS-PAS-HIG-16-022 (2016).

[9] The CMS Collaboration, CMS-PAS-HIG-16-019 (2016).

[10] The CMS Collaboration, CMS-PAS-HIG-16-031 (2016). 
[11] The CMS Collaboration, CMS-PAS-HIG-16-037 (2016).

[12] The CMS Collaboration, CMS-DP2016-064 (2016).

[13] The CMS Collaboration, CMS-TDR-15-02 (2016). 